\title{
IgG4-related Dacryoadenitis and Sialadenitis with Palatal Gland Swelling
}

\author{
Masayasu Yoshida ${ }^{1,2)}$, Akira Baba ${ }^{3)}$, Kazuhiko Hashimoto ${ }^{4)}$, \\ Yuriko Nakamura ${ }^{2)}$, Yasutaka Itou ${ }^{2)}$, Yoshifumi Yoshida ${ }^{2)}$, \\ Kazumichi Sato ${ }^{2)}$ and Takeshi Nomura ${ }^{2)}$ \\ ${ }^{1)}$ Department of Oral and Maxillofacial Surgery, Yokohama Minami Mutual Hospital, \\ 1-21-1 Mutsuurahigashi, Kanazawa-ku, Yokohama, Kanagawa 236-0037, Japan \\ 2) Department of Oral Medicine, Oral and Maxillofacial Surgery, Tokyo Dental College, \\ 5-11-13 Sugano, Ichikawa, Chiba 272-8513, Japan \\ ${ }^{3)}$ Department of Radiology, The Jikei University School of Medicine, \\ 3-19-18 Nishi-Shimbashi, Minato-ku, Tokyo 105-0003, Japan \\ ${ }^{4)}$ Division of Surgical Pathology, Clinical Laboratory, Ichikawa General Hospital, Tokyo \\ Dental College, \\ 5-11-13 Sugano, Ichikawa, Chiba 272-8513, Japan
}

Received 8 January, 2020/Accepted for publication 28 August, 2020

Published Online in J-STAGE 15 February, 2021

\begin{abstract}
Many cases of immunoglobulin G4-related disease (IgG4-RD) involve swelling of the salivary glands, often affecting the parotid and submandibular glands in the head and neck region, in addition to the lacrimal glands. Cases of IgG4-RD characterized by swelling of the palatal glands, however, are extremely rare. Here, we describe a case of IgG4$\mathrm{RD}$ involving bilateral swelling of the palatal glands. An 83-year-old man presented at our hospital with bilateral swelling of the lacrimal and submandibular glands. A solid bilateral swelling of normal colour and unclear boundaries was observed in the hard palate. The lesion was diagnosed as IgG4-RD and steroid therapy initiated. A subsequent decrease in swelling of the palatal, lacrimal, and submandibular glands was confirmed by magnetic resonance imaging. These results indicate that dentists should suspect IgG4-RD in patients presenting with bilateral swelling of the palatal glands.
\end{abstract}

Key words: IgG4-related dacryoadenitis and sialadenitis-

Palatal gland swelling — Magnetic resonance imaging

\section{Introduction}

The etiology of immunoglobulin G4-related disease (IgG4-RD) is unknown. It is characterized by general swelling of the organs, including the lacrimal and salivary glands, the pres- 


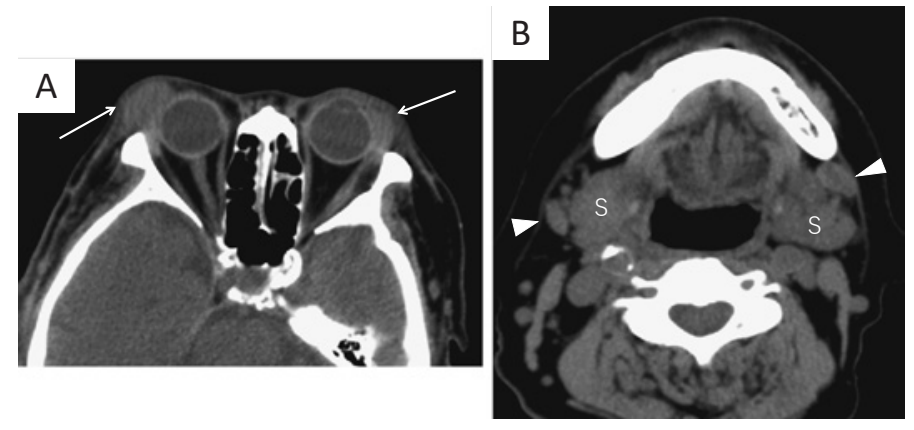

Fig. 1 Observations from computed tomography prior to treatment A: Bilateral lacrimal gland swelling (arrow), B: Bilateral submandibular gland swelling (S), and bilateral multiple lymph node enlargement (arrowhead) were visible

ence of nodular/hypertrophic lesions due to lymphocytic infiltration, IgG4-positive plasma cell infiltration, and fibrosis. It has been reported that this disease can help in differentiating autoimmune pancreatitis from pancreatic cancer, as IgG4 levels are significantly higher in the former ${ }^{3)}$. One study found IgG4positive plasma cells in excised pancreas ${ }^{4)}$, establishing a new concept of IgG4-RD. In addition to swelling of the salivary glands, some recent studies have also reported labial swelling ${ }^{5)}$. Here, we report a rare case of IgG4$\mathrm{RD}$ involving swelling of the palatal glands.

\section{Case Presentation}

The patient was an 83-year-old man who initially visited the Department of Ophthalmology at our hospital with swelling of the right eyelid. This had been left untreated for approximately 1 year before seeking medical advice, as it had been unaccompanied by any other acute symptoms. He had only decided to seek medical help when the other eyelid also started to show signs of gradual swelling. Visual examination revealed swelling in the head and neck region. Subsequent computed tomography (CT) revealed the following: (i) swelling of the bilateral lacrimal and submandibular glands; and (ii) multiple lymph node enlargement in the bilateral submandibular regions. No neoplastic disease or sialolithiasis was observed originating in the submandibular glands (Figs. 1A, 1B). The patient had a history of hypertension, gastric ulcer, bronchial asthma, and cataracts. Therefore, he was referred to us at the Department of Oral Medicine, Oral and Maxillofacial Surgery. At the first visit, bilateral swelling of the eyelids and submandibular glands was observed (Fig. 2A). Although there was no obvious swelling of the floor of the mouth, a solid swelling of normal colour and unclear boundaries was observed in the bilateral hard palate (Fig. 3A). Panoramic radiography to evaluate swelling of the palate revealed no abnormalities in the teeth or jawbone. No labial swelling was observed. Stimulated salivary flow rate in the gum test was $25 \mathrm{ml} / 10 \mathrm{~min}$. An IgG4-RD was suspected, so a blood test was performed. The results showed that the serum amylase level (112 IU/liter) was below the threshold, whereas the white blood cell count $(11,700 /$ $\mathrm{m}^{2}$ ) and total IgG and serum IgG4 levels were above their respective thresholds (Table 1 ). Magnetic resonance imaging revealed bilateral swelling of the submandibular, parotid, and lacrimal glands. Short-TI inversion recovery imaging revealed a somewhat hyperintense bilateral submandibular gland and hypointense structures on both sides of the hard palate on T2-weighted images (Fig. 4A). Thoracoabdominal CT revealed bilateral pleural effusions with pleural thickening, suggesting pleuritis. It also revealed circumferen- 

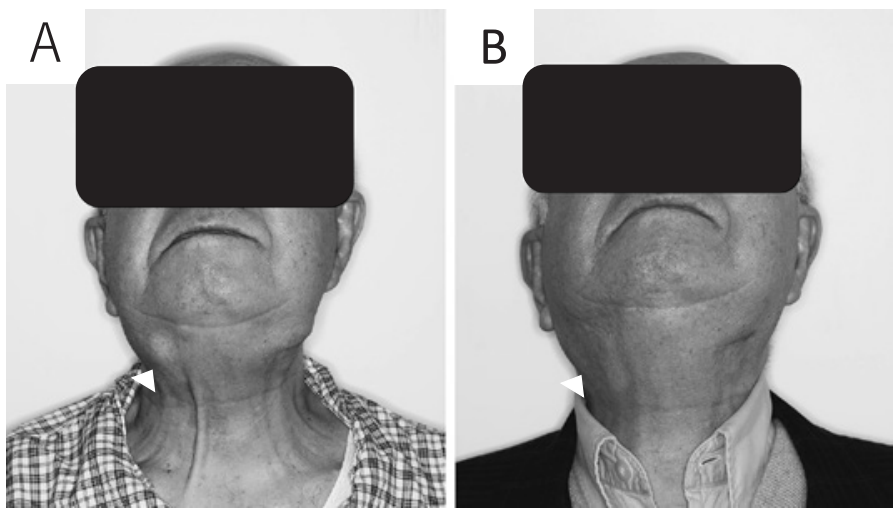

Fig. 2 Facial expression images before and after treatment

A: Facial observation of submandibular gland swelling before steroid therapy. B: Facial observation showing disappearance of submandibular gland swelling after steroid therapy.
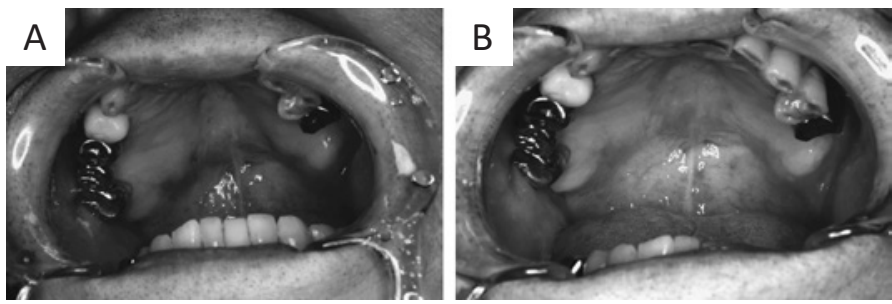

Fig. 3 Intraoral observations before and after treatment

A: Diffuse swelling of palate in bilateral maxillary molar areas before steroid therapy. B: Healing of diffuse swelling in bilateral palate after steroid therapy.

Table 1 Blood test findings at the first visit

\begin{tabular}{lc}
\hline \hline Inspection item & Measured value \\
\hline $\mathrm{RBC}$ & $460 \times 10 / \mu \mathrm{l}$ \\
$\mathrm{Hb}$ & $12.9 \mathrm{~g} / \mathrm{dl}$ \\
$\mathrm{WBC}$ & $11700 / \mu \mathrm{l}$ \\
$\mathrm{ALB}$ & $3.4 \mathrm{~g} / \mathrm{dl}$ \\
$\mathrm{Amylase}$ & $112 \mathrm{IU} / \mathrm{liter}$ \\
$\mathrm{BUN}$ & $23.1 \mathrm{mg} / \mathrm{dl}$ \\
$\mathrm{Cre}$ & $0.88 \mathrm{mg} / \mathrm{dl}$ \\
$\mathrm{AST}$ & $28 \mathrm{IU} / \mathrm{liter}$ \\
$\mathrm{ALT}$ & $29 \mathrm{IU} / \mathrm{liter}$ \\
$\gamma \mathrm{GTP}$ & $18 \mathrm{IU} / \mathrm{liter}$ \\
$\mathrm{IgG}$ & $2,983 \mathrm{mg} / \mathrm{dl}$ \\
$\mathrm{IgG} 4$ & $1,220 \mathrm{mg} / \mathrm{dl}$ \\
\hline
\end{tabular}

tial thickening of the gallbladder wall, suggesting chronic cholecystitis.

\section{Clinical Procedures and Outcomes}

The results of the blood test revealed high serum IgG4 levels. Therefore, taking the pathogenesis of IgG4-RD into consideration, the patient was offered consultation liaison services with the Department of Internal Medicine (Rheumatology) at our hospital. In the meanwhile, chronic multiple lymphadenopathies were observed in the bilateral submandibular space together with swelling of the submandibular glands. A biopsy of a swollen lymph node in the submandibular region 

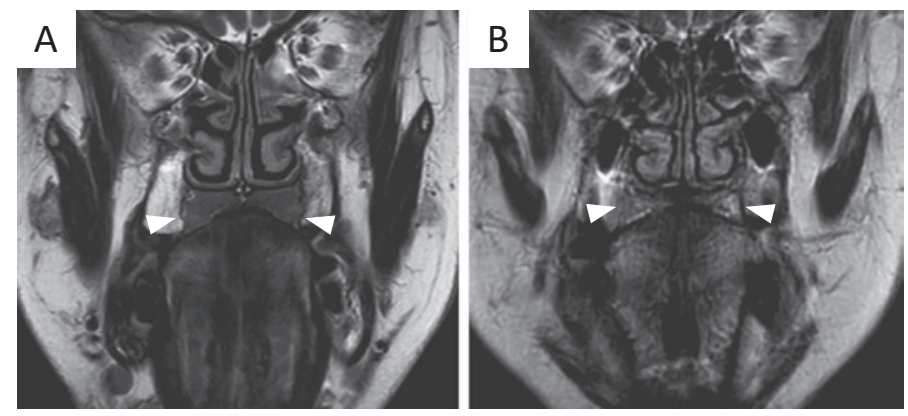

Fig. 4 Palate before and after treatment (T2-weighted image, coronal section)

A (before treatment), B (after treatment): Bilateral palate showing no significant tumour-like structures but significant shrinking tendency versus pre-treatment.

was performed under local anaesthesia. Haematoxylin and eosin staining revealed severe lymphocyte infiltration with clear formation of large and small lymphoid follicles in the germinal centre. Although the follicular structure was maintained (Fig. 5A), interfollicular and intrafollicular plasma cell infiltrations were observed (Fig. 5B). Under immunostaining, the lymph node structure showed a tendency toward positive for CD3, CD5, CD20, and CD79a, while the lymph follicles, which exhibited bel-2 (-) and CD10 (+), tested negative for malignant lymphoma. Many cells showed infiltration of IgG4 plasma cells. Most plasma cells were IgG4-positive (Figs. 5C, 5D). Based on the aforementioned clinical/hematologic/histopathological findings, the definitive diagnosis was IgG4-RD.

The patient was prescribed $15 \mathrm{mg} /$ day prednisolone at the Department of Internal Medicine (Rheumatology). Subjective and objective improvement in lacrimal and submandibular gland swelling were observed at approximately 1 month later (Fig. 2B). The patient also reported a subjective increase in oral cavity volume (Fig. 3B). Magnetic resonance imaging at 3 months after commencement of steroid therapy revealed a tendency toward shrinkage in both the bilateral submandibular and lacrimal glands, as well as in the hypointense structures observed on both sides of the hard palate (Fig. 4B). The steroid dosage was gradually reduced to a maintenance dosage of $5 \mathrm{mg} /$ day. A good clinical course was observed with no recurrence of symptoms.

\section{Discussion}

Systemic in nature, IgG4-RDs can lead to single, as well as multiple organ dysfunction syndrome (e.g., pancreas, salivary, pituitary, and thyroid glands, lungs, retroperitoneum, kidney, and prostate). Clinically, symptoms vary depending on the extent of the lesions and which organ is involved. Therefore, disease can sometimes be accompanied by the development of severe complications such as organomegaly, compression symptoms, cell infiltration, obstruction due to tissue hypertrophy or fibrosis, and related organ failure. Treatment may be ineffective in cases showing fibrotic change due to chronic infiltration, leading to irreversible organ damage. An autoimmune mechanism seems to be involved in IgG4-RD, and steroid therapy is often the initial treatment of choice. Early intervention is crucial in preventing irreversible damage, so early diagnosis is crucial.

Swelling in the lacrimal and submandibular glands persisting for over 3 months was taken into account in arriving at a diagnosis in the present case. The usefulness of echogra- 

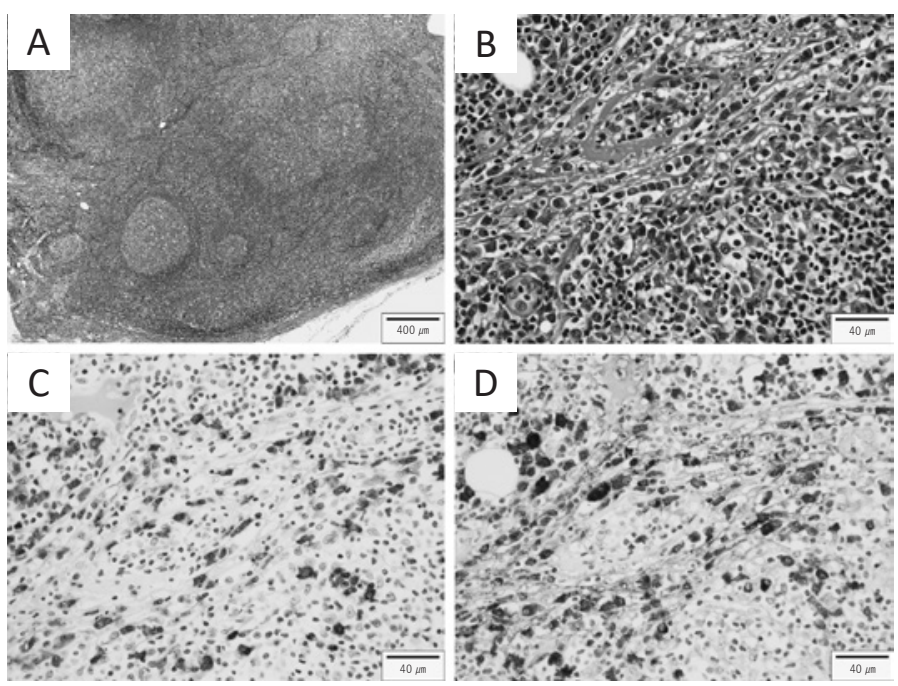

Fig. 5 Histopathological findings of lymph node biopsy

A, B: Hematoxylin and eosin staining, C: Anti-immunoglobulin G ( $\operatorname{IgG}$ ) antibody, D: Anti-IgG4 antibody Lymphoid tissue showing clear formation of large and small lymphoid follicles in germinal centre $(\mathrm{A}, 40 \times)$. Plasma cell infiltration was observed in stroma between lymphoid follicles. (B, $400 \times)$.

On immunostaining, IgG-positive plasma cell infiltration was visible, and most samples showed IgG4 positivity $(\mathrm{C}, \mathrm{D}, 400 \times)$.

phy or sonography in screening for IgG4-RD has been reported in a case of submandibular swelling $^{12}$. Neither imaging modality was used in the present case, however. Serologically elevated serum IgG4 levels, histopathologically significant lymphocyte/plasma cell infiltration, an IgG4/IgG-positive cell ratio $\geq 40$ $\%$, and IgG4-positive plasma cells $(>10 /$ high power field) were observed. A biopsy of the significantly swollen submandibular lymph nodes was performed. An IgG4-RD is likely to be complicated by the development of malignant tumors or lymphomas ${ }^{17,18}$. This indicates that a biopsy of the swollen lymph nodes in such patients is warranted.

Clinical swelling in the head and neck region in patients with IgG4-RD involves the submandibular, parotid, and lacrimal glands ${ }^{1,7)}$. Approximately $70 \%$ of patients with IgG4-related kidney disease show symptoms of salivary gland swelling ${ }^{6)}$. As for the minor salivary glands, in one earlier study, a biopsy of a swollen lip revealed IgG4-positive plasma cell infiltration in the labial glands, resulting in a diagnosis of IgG4-RD with elevated serum IgG4 levels ${ }^{5}$. In another study, the palatal and labial glands were swollen in 20 cases of Mikulicz disease ${ }^{10)}$. In one case report of IgG4-RD with palatal gland swelling ${ }^{2}$, where the patient declined corticosteroid treatment, the swelling of the palatal glands remained stable after presentation. To our knowledge, the present case of IgG4-RD is the first to compare imaging findings between before and after treatment. A search of the literature revealed no other report of lingual, buccal, or molar salivary gland swelling in IgG4-RD, and the pathogenesis of tissue selectivity in the affected salivary gland remains unknown. However, many studies recommend a biopsy of the submandibular glands for a more accurate diagnosis ${ }^{9,13}$, as the minor salivary glands can also be affected. At present, some patients may require more accurate evaluation of minor salivary gland swelling, as diagnosis is mainly based on evaluation of the major sali- 
vary glands, where significant swelling is usually observed. Case reports on the relationship between IgG4-RD and swelling of the minor salivary glands demonstrate the benefit of minimally invasive diagnostics in such cases. Although no biopsy of the palate was performed in the present case, palatal swelling was considered to indicate IgG4-RD based on the clinical course and the imaging findings.

Recently, many basic and clinical studies have contributed to the elucidation of the pathophysiology of IgG4-RD. Steroid therapy is usually the first choice of treatment for this disease. However, a certain number of patients have refractory IgG4-RD due to dose reduction or discontinuation ${ }^{15)}$. In one study, the molecular-targeted drug Rituximab was effective in patients with refractory $\operatorname{IgG} 4-\mathrm{RD}^{14)}$. Since Rituximab improves clinical symptoms by depleting $\mathrm{B}$ cells, the interaction between IgG4-positive plasmablasts and CD4 + T cells has been suggested to be at the core of the pathogenesis of IgG4-RD ${ }^{8}$. In addition, the efficacy of the biological agent Abatacept on IgG4-RD and the therapeutic application of Elotuzumab $^{11,16)}$ in the treatment of multiple myeloma have been studied ${ }^{14)}$. Although various therapies are being studied in this manner, early diagnosis remains one of the most important factors in controlling the pathological conditions of this disease. The results of the present case indicate the need to suspect IgG4-RD in patients presenting with swollen lesions of the bilateral palatal glands.

\section{Acknowledgements}

We are grateful to Dr. Noriyuki Seta of the Department of Internal Medicine (Rheumatology), Tokyo Dental College, Ichikawa General Hospital, for helpful discussions and treatment of the patient.

There is no conflict of interest to declare. No funding was acquired for this study.

\section{Ethical Approval}

All procedures performed were in accordance with the ethical standards of the institutional and/or national research committee and with the 1964 Helsinki declaration and its later amendments or comparable ethical standards. Written informed consent was obtained from the patient for inclusion in this report.

\section{References}

1) Abe T, Kobayashi A, Imai K, Sakata $Y$, Sato $T$, Yoda T (2008) A case of IgG4-related chronic sclerosing sialadenitis associated with autoimmune pancreatitis. Nihon Koukuugeka Gakkai Zasshi 54:558-562. (in Japanese)

2) Andrew N, Kearney D, Sladden N, Goss A, Selva D (2014) Immunoglobulin G4-related disease of the hard palate. Nihon Koukuugeka Gakkai Zasshi 72:717-723. (in Japanese)

3) Hamano H, Kawa S, Horiuchi A, Unno H, Furuya N, Akamatsu T, Fukushima M, Nikaido T, Nakayama K, Usuda N, Kiyosawa K (2001) High serum IgG4 concentrations in patients with sclerosing pancreatitis. N Engl J Med 344: 732-738.

4) Hamano H, Kawa S, Ochi Y, Unno H, Shiba N, Wajiki M, Nakazawa K, Shimojo H, Kiyosawa K (2002) Hydronephrosis associated with retroperitoneal fibrosis and sclerosing pancreatitis. Lancet 359:1403-1404.

5) Kato S, Enokida Y, Sato H, Shimizu H, Fujita K, Endo T, Nakagawa T, Tsunoda K (2016) IgG4related dacryoadenitis and sialadenitis associated with minor salivary gland swelling; a case report. Nihon Koukuunaika Gakkai Zasshi 22: 89-94. (in Japanese)

6) Kawano M, Saeki T, Nakashima H, Nishi S, Yamaguchi Y, Hisano S, Yamanaka N, Inoue D, Yamamoto M, Takahashi H, Nomura H, Taguchi T, Umehara H, Makino H, Saito T (2011) Proposal for diagnostic criteria for IgG4-related kidney disease. Clin Exp Nephrol 15:615-626.

7) Kodaka R, Yago K, Yamada M, Kameyama K, Nakagawa T, Asanami S (2011) A case of IgG4related chronic sclerosing sialadenitis with suspected transition to Mikulicz's disease. Nihon Koukuugeka Gakkai Zasshi 57:476-480. (in Japanese)

8) Maehara T (2017) IgG4-related disease-Mechanistic insights from both clinical and immu- 
nologic understanding of this condition. Nihon Rinsho Meneki Gakkai Kaishi 40:206212. (in Japanese)

9) Moriyama M, Furukawa S, Kawano S, Goto Y, Kiyoshima T, Tanaka A, Maehara T, J-N Hayashida N, Ohta M, Nakamura S (2014) The diagnostic utility of biopsies from the submandibular and labial salivary glands in IgG4related dacryoadenitis and sialoadenitis, socalled Mikulicz's disease. Int J Oral Maxillofac Surg 43:1276-1281.

10) Moriyama M, Tanaka A, Maehara T, Ohyama Y, Shimizu A, Nakashima H, Hayashida J, Shinozaki S, Kubo Y, Furukawa S, Kikuta T, Nakamura S (2013) Clinical characteristics of Mikulicz's disease as an IgG4-related disease. Clin Oral Invest 17:1995-2002.

11) Pomponio G, Olivari D, Mattioli M, Angeletti A, Rossetti G, Goteri G, Gabrielli A (2018) Sustained clinical response after single course of rituximab as first-line monotherapy in adult-onset asthma and periocular xanthogranuloma syndrome associated with IgG4related disease: A case report. Medicine (Baltimore) 97: e11143.

12) Shimizu M, Okamura K, Kise Y, Takeshita Y, Furuhashi W, Weerawanich W, Moriyama M, Ohyama Y, Furukawa S, Nakamura S, Yoshimura K (2015) Effectiveness of imaging modalities for screening IgG4-related dacryoadenitis and sialadenitis (Mikulicz's disease) and for differentiating it from Sjögren's syndrome (SS), with an emphasis on sonography. Arth ritis Res Ther 17:223.

13) Takano K, Nomura K, Abe A, Kamekura R, Yamamoto M, Ichimiya S, Takahashi H, Himi $\mathrm{T}$ (2016) Clinicopathological analysis of salivary gland tissue from patients with IgG4related disease. Acta Otolaryngol 136: $717-721$.
14) Topazian M, Witzig TE, Smyrk TC, Pulido JS, Levy MJ, Kamath PS, Chari ST (2008) Rituximab therapy for refractory biliary strictures in immunoglobulin G4-associated cholangitis. Clin Gastroenterol Hepatol 6: 364-366.

15) Umehara H, Okazaki K, Masaki Y, Kawano M, Yamamoto M, Saeki T, Matsui S, Yoshino T, Nakamura S, Kawa S, Hamano H, Kamisawa T, Shimosegawa T, Shimatsu A, Nakamura S, Ito T, Notohara K, Sumida T, Tanaka Y, Mimori T, Chiba T, Mishima M, Hibi T, Tsubouchi H, Inui K, Ohara H (2012) Comprehensive diagnostic criteria for IgG4-related disease (IgG4-RD), 2011. Mod Rheumatol 22:21-30.

16) Yamamoto $M$ (2016) New strategies for the treatment of IgG4-related disease. Nihon Rinsho Meneki Gakkai Kaishi 39:485-490. (in Japanese)

17) Yamamoto M, Takahashi H, Tabeya T, Suzuki C, Naishiro Y, Ishigami K, Yajima H, Shimizu Y, Obara M, Yamamoto H, Himi T, Imai K, Shinomura Y (2012) Risk of malignancies in IgG4-related disease. Mod Rheumatol 22: 414-418.

18) Zintzaras E, Voulgarelis M, Moutsopoulos HM (2005) The risk of lymphoma development in autoimmune diseases: a meta-analysis. Arch Intern Med 165:2337-2344.

\section{Correspondence:}

Dr. Masayasu Yoshida

Department of Oral and

Maxillofacial Surgery,

Yokohama Minami Mutual Hospital, 1-21-1 Mutsuurahigashi, Kanazawa-ku, Yokohama, Kanagawa 236-0037, Japan E-Mail: qoo031373@gmail.com 